\title{
Indirect Decompression Using Lateral Lumbar Interbody Fusion for Restenosis after an Initial Decompression Surgery
}

\author{
Hiroaki Nakashima ${ }^{1}$, Tokumi Kanemura ${ }^{1}$, Kotaro Satake ${ }^{1}$, Kenyu Ito $^{1}$, Yoshimoto Ishikawa ${ }^{1}$, \\ Jun Ouchida ${ }^{1}$, Naoki Segi ${ }^{2}$, Hidetoshi Yamaguchi ${ }^{2}$, Shiro Imagama ${ }^{2}$ \\ ${ }^{1}$ Department of Orthopedic Surgery, Konan Kosei Hospital, Konan, Japan \\ ${ }^{2}$ Department of Orthopedic Surgery, Nagoya University Graduate School of Medicine, Nagoya, Japan
}

\begin{abstract}
Study Design: Retrospective comparative study.
Purpose: We compared clinical and radiographical outcomes after lumbar decompression revision surgery for restenosis by lateral lumbar interbody fusion (LLIF) and posterior lumbar interbody fusion (PLIF).

Overview of Literature: Indirect lumbar decompression with LLIF was used to treat degenerative lumbar diseases requiring neural decompression. However, only a few studies have focused on the effectiveness of this technique for restenosis after lumbar decompression.

Methods: We retrospectively investigated 52 cases involving lumbar interbody fusions for restenosis with spondylolisthesis after lumbar decompressions; these cases consisted of 15 patients who underwent indirect decompression with LLIF and posterior fixation and 37 patients who underwent the same procedure with PLIF. We compared Japanese Orthopaedic Association (JOA) scores and perioperative complications between groups. The cross-sectional areas of the thecal sac on magnetic resonance imaging were measured before, immediately after, and 2 years after surgery. We conducted statistical analyses using unpaired $t$-test and Fisher's exact tests, and a $p$-value $<0.05$ was considered statistically significant.

Results: The operative time was significantly shorter in the LLIF group than in the PLIF group $(115.3 \pm 33.6$ min vs. $186.2 \pm 34.2 \mathrm{~min}$, respectively; $p<0.001$ ). In addition, the intraoperative blood loss was significantly lower in the LLIF group than in the PLIF group (58.2 \pm 32.7 $\mathrm{mL}$ vs. $303.2 \pm 140.1 \mathrm{~mL}$, respectively; $p<0.001)$. We found two cases of transient lateral thigh weakness (13.3\%) in the LLIF group and five cases of incidental durotomy, one case of deep infection, and one case of neurological deterioration in the PLIF group —resulting in a higher complication incidence (18.9\%), although it did not reach ( $p=0.63)$. The JOA scores improved significantly in both groups. Conclusions: Indirect decompression using LLIF provided acceptable clinical and radiographical outcomes in patients with restenosis with spondylolisthesis after lumbar decompression; no revision-surgery-specific complications were found. Our results suggest that LLIF is a safe and minimally invasive procedure for revision surgery.
\end{abstract}

Keywords: Indirect decompression; Lateral lumbar interbody fusion; Reoperation; Posterior lumbar interbody fusion; Dural tear

Received Jun 12, 2019; Revised Jul 2, 2019; Accepted Jul 4, 2019

Corresponding author: Hiroaki Nakashima

Department of Orthopedic Surgery, Konan Kosei Hospital, 137 Takayamachi Omatsubara, Konan 483-8704, Japan

Tel: +81-587-51-3333, Fax: +81-587-51-3300, E-mail: hirospine@gmail.com 


\section{Introduction}

Posterior lumbar interbody fusion (PLIF) and transforaminal lumbar interbody fusion (TLIF) have been used in the treatment of restenosis with spondylolisthesis after lumbar decompression surgery. Although PLIF and TLIF provide good clinical outcomes, complications such as incidental durotomy, nerve injury, massive blood loss, and epidural hematoma occur frequently with direct decompression in patients with revision surgery because of operative scar formation after the primary procedure [17].

Indirect decompression using lateral lumbar interbody fusion (LLIF) has been used during primary surgery for lumbar spondylolisthesis as an alternative to PLIF/TLIF [8]. LLIF is a minimally invasive lumbar fusion technique with excellent surgical outcomes. LLIF allows maximal disk resection and endplate preparation by approaching disks from the lateral side and results in indirect spinal canal decompression when the large interbody cage ligament taxis technique is used [9-11]. Significant radiographical improvements have been addressed in several reports, including $41.9 \%-89.0 \%$ increases in disk height, $13.5 \%-38.0 \%$ increases in foraminal height, $35.0 \%-69.0 \%$ increases in foraminal areas, and $30.2 \%-43.0 \%$ increases in thecal sac areas $[9,11,12]$.

However, only a few studies have addressed the efficacy of indirect decompression using LLIF in revision cases, which may be complicated by operative scars. Using LLIF combined with percutaneous pedicle screw (PPS) insertion, the thecal sac can be decompressed without having to deal with operative scars. Thus, we hypothesized that this surgical method can be applied during revision operations achieving sufficient decompression and good clinical outcomes using indirect decompression to minimize perioperative complications.

We have achieved challenging indirect decompressions using LLIF in revision cases with spondylolisthesis after lumbar decompression surgery. For this study, our aim was to investigate the perioperative complications, surgical outcomes, and clinical recovery in revision cases using indirect decompression and by comparing differences with direct decompression using PLIF.

\section{Materials and Methods}

We conducted this retrospective single-center study by reviewing the surgical and radiographic data of patients diagnosed to have degenerative spondylolisthesis with restenosis who underwent lumbar decompressions and surgical intervertebral fusions. Our institutional review board approved the study. This study is a retrospective study, and so informed consent was not obtained from each subject. In this study, we included patients with revision surgery at the same level as that in the initial surgery. All patients underwent single-level fusion surgery. We excluded patients with lumbar disk herniation, L5-S fusion, neurological diseases, spondylolisthesis grade >II (50\%), or fusion level $>1$.

The final study sample consisted of 52 consecutive patients (from January 2008 to March 2017 at institution) followed up for more than 2 years after surgery: 37 patients with PLIF for spondylolisthesis and restenosis after lumbar decompression and 15 patients with LLIF and posterior fixation. Indirect decompressions were performed in patients without severe neurological symptoms (such as paralysis or severe leg pain at rest) and in those without a free disk fragment on magnetic resonance imaging (MRI). We started performing indirect decompressions using LLIF in this institution in 2013; before that, only PLIFs were performed. The TLIF technique was not used in any of the cases in this study. All patients included in the study who were operated on after 2013 underwent indirect decompressions using LLIF, except for one patient who had severe leg pain at rest.

\section{Surgical details of lateral lumbar interbody fusion}

Of the 15 patients who underwent LLIF, 13 underwent extremely lateral lumbar fusions (XLIFs) using Coroent cages (NuVasive Inc., San Diego, CA, USA), whereas two underwent oblique lumbar interbody fusions (OLIFs) using Clydesdale cages (Medtronic, Minneapolis, MN, USA). We approached the intervertebral space directly through the psoas muscle in cases with XLIF and between the psoas muscle and the aorta in patients with OLIF [8]. All patients with XLIF were subjected to neuromonitoring.

The patients were placed in the lateral decubitus position, with slight hip and knee flexion (approximately $30^{\circ}$ ) on a "breakable" surgical table. The LLIFs were performed with intraoperative fluoroscopy using either a trans-psoas approach or passing between the psoas muscle and the aorta. After disk resection, the cage trials and insertions were performed under fluoroscopy guidance. 
After the LLIF procedure, the patients were repositioned in the prone position. Posterior lumbar fixations using PPSs were then performed using an O-arm-based navigation system. The reference of the navigation system was placed at the cranial spinous process.

\section{Clinical assessments}

Japanese Orthopaedic Association (JOA) neurological scores were evaluated before surgery, as well as 6 months and 2 years after surgery. In addition, we collected information on perioperative surgical complications.

\section{Radiographical assessments}

MRIs scans were obtained before the procedure and 2 weeks after the operations. The cross-sectional area of the thecal sac was measured using T2-weighted axial MRI at the same time points.

\section{Statistical analysis}

We used Student $t$-test and chi-square test to compare the years versus between the two groups. A $p$-value of $<0.05$ was considered statistically significant. All analyses were conducted using the IBM SPSS ver. 21.0 software (IBM Corp., Armonk, NY, USA).

\section{Results}

We found no significant differences in mean age $(73.6 \pm 4.8$ years versus $75.2 \pm 6.3$ years), gender (male/female ratios, 23:14 versus 9:6), or number of fused disk levels between the groups (Table 1). The operative time was significantly shorter in the LLIF group than in the PLIF group $(115.3 \pm 33.6$ minutes versus $186.2 \pm 34.2$ minutes, respectively; $p<0.001)$. In addition, the intraoperative blood loss was significantly lower in the LLIF group than in the PLIF group $(58.2 \pm 32.7 \mathrm{~mL}$ versus $303.2 \pm 140.1 \mathrm{~mL}$, respectively; $p<0.001)$.

As for perioperative complications, we found two patients with transient thigh weakness (13.3\%) in the LLIF group, but they all recovered within 1 month after the operation. By contrast, the perioperative complication rate was higher (although it did not reach statistical significance; $p=0.63$ ) in the PLIF group (18.9\%), with such complications as incidental durotomy (five cases), deep infection (one case), and neurological deterioration (one case).

\section{Improvement of thecal sac area on magnetic reso- nance imaging}

The mean preoperative cross-sectional areas of the thecal sac were $53.5 \pm 33.1$ and $61.3 \pm 21.4 \mathrm{~mm}^{2}$ in the PLIF and LLIF groups $(p=0.40$ ), respectively. At 2 weeks after surgery, this thecal sac area had improved to $146.2 \pm 34.7$ $\mathrm{mm}^{2}$ (238.5\% of the preoperative value) in the PLIF group and to $85.7 \pm 21.2 \mathrm{~mm}^{2}$ (160.2\% of the preoperative value) in the LLIF group $(p<0.001)$. The thecal sac areas further improved from 2 weeks after surgery onward in the LLIF group, reaching $115.7 \pm 31.3 \mathrm{~mm}^{2}$ (216.2\% of the preoperative value) 2 years after the operation.

\section{Japanese Orthopaedic Association scores}

The preoperative JOA scores were $10.1 \pm 4.2$ in the PLIF

Table 1. Patients' demographic data

\begin{tabular}{lccc} 
Characteristic & Posterior lumbar interbody fusion & Lateral lumbar interbody fusion & $p$-value \\
\hline No. of cases & 37 & 15 & \\
\hline Age (yr) & $73.6 \pm 4.8$ & $75.2 \pm 6.3$ & 0.33 \\
\hline Male/female & $23 / 14$ & $9 / 6$ & 0.88 \\
\hline No. of disc level operated on & & & 0.97 \\
\hline L2-3 & 2 & 1 & \\
\hline L3-4 & 14 & 8 & 0.87 \\
\hline L4-5 & 21 & $10.3 \pm 2.7$ & \\
\hline Preoperative Japanese Orthopaedic Association score & $10.1 \pm 4.2$ & & \\
\hline
\end{tabular}

Values are presented as number or mean \pm standard deviation. 
group and $10.3 \pm 2.7$ in the LLIF group $(p=0.87)$. At 6 months after surgery, the scores had improved to $21.8 \pm 4.5$ in the PLIF group and to $22.2 \pm 4.4$ in the LLIF group $(p=0.77)$. Moreover, these JOA scores remained stable at 2 years after surgery $(21.8 \pm 4.6$ and $21.9 \pm 4.2$ in the PLIF and LIF+PPS groups, respectively; $p=0.94$ ). We found no statistically significant differences between the two groups at any time point.

\section{Representative case}

A 68-year-old man presented with recurrent bilateral buttock and lower limb pain 5 years after undergoing lumbar decompression surgery at L4-L5. The radiographical examination showed lumbar spinal stenosis at L4-L5 with L4 degenerative spondylolisthesis (Fig. 1A-C). Indirect decompression using LLIF and PPSs was performed at L4-L5 (Fig. 1D). The patient's preoperative symptoms markedly improved after the procedure. After 2 weeks, his thecal sac areas on MRI were significantly larger (122.1 $\left.\mathrm{mm}^{2}\right)$ compared with those recorded preoperatively $(84.7$ $\mathrm{mm}^{2}$ ) (Fig. 1E, F).

\section{Discussion}

Our results suggest that indirect lumbar decompression
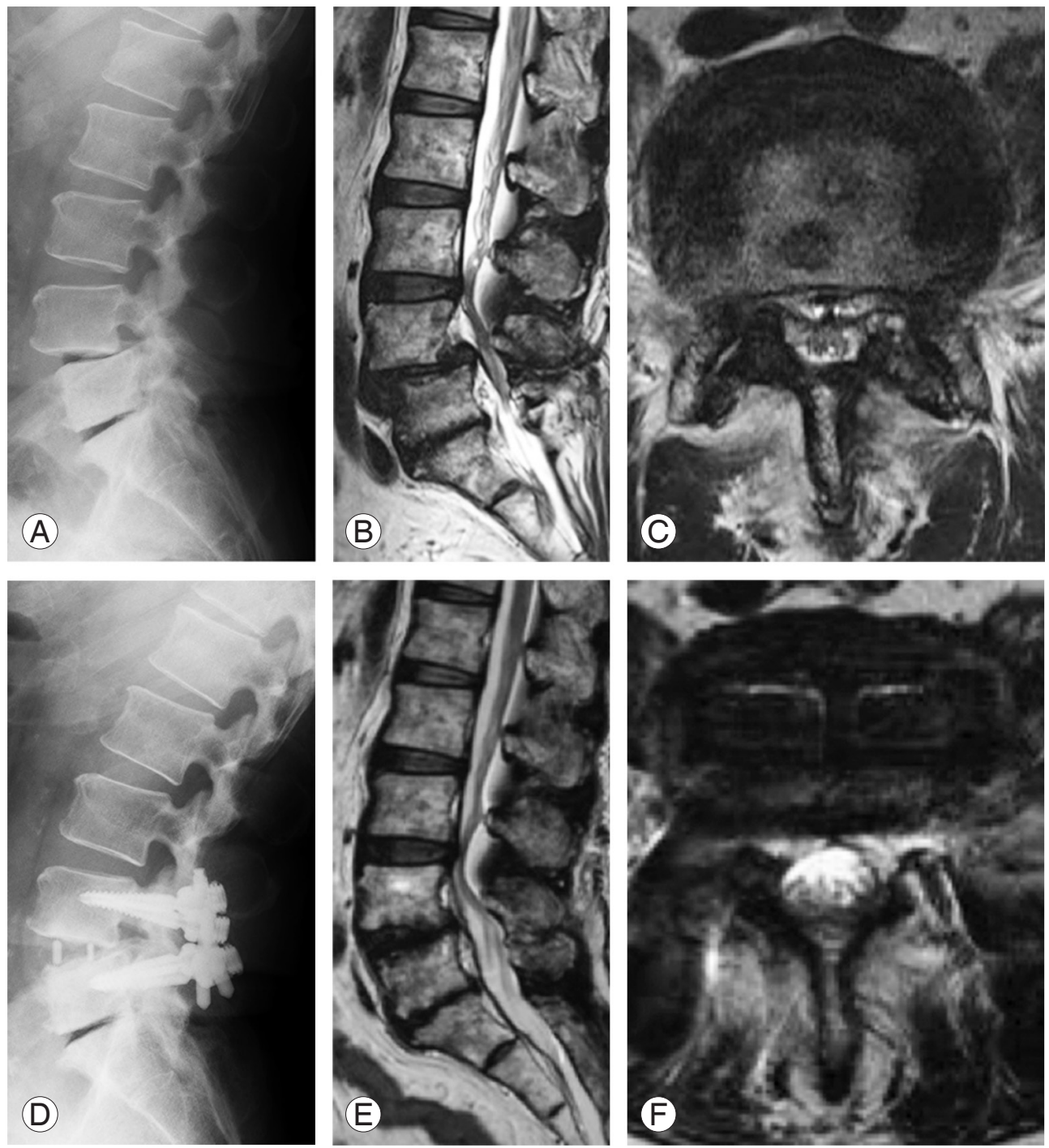

Fig. 1. Representative images of a 68-year-old male patient with $L 4$ spondylolisthesis. (A) Preoperative lateral X-ray image. (B, C) Preoperative MRI images. (D) Postoperative lateral X-ray view. (E, F) Postoperative axial MRI image at $\mathrm{L} 4-\mathrm{L} 5$ disk level 2 weeks after the operation. MRI, magnetic resonance imaging. 
using LLIF and posterior instrumentation minimizes perioperative complications specific to revision surgery and provides appropriate clinical and radiographical recoveries similar to those in cases where PLIF is used. These preliminary findings suggest that indirect decompression is effective and relatively safe when used during primary and revision procedures.

Revision surgery after lumbar decompression remains a challenging procedure for spine surgeons [3-6]. A surgical scar is formed around the area operated on, making it difficult to identify anatomical landmarks, and incidental durotomies often occur because of adhesions between the dura and the surgical scar. Incidental durotomies occur approximately 2 to 5 times more often during revision surgeries than during primary surgeries, and nerve injury and neurological deterioration may occur as consequences [3-6]. In addition, surgical complications such as deep infections often occur during revision surgeries, resulting in long hospital stays and high medical costs [7]. For these reasons, it has been an important goal to find ways to minimize perioperative complications during revision surgeries.

To avoid dealing with surgical scars, we started to apply indirect decompression during both primary and revision surgeries. LLIF was developed as a minimally invasive lumbar interbody fusion technique, and this technique has been adopted for the treatment of axial lower back pain, spondylolisthesis, spinal stenosis, and adult scoliosis [8,11,13-16]. Compared with PLIF, LLIF offers advantages derived from the insertion of a large interbody cage from the lateral side of the spine $[8,17]$. This large interbody cage allows indirect decompression via sufficient traction of the disk space, foramen, and hypertrophied ligament. Several reports have highlighted significant improvements in such radiographic parameters as disk height, foraminal height, foraminal area, and thecal sac area after indirect decompression using LLIF, and they have presented data indicating sufficient clinical improvement after the procedure $[9,11,12]$.

However, only few reports have focused on indirect decompression adopted for revision cases, and the clinical outcomes and complications of this operation were unclear. For example, Hayama et al. [18] investigated the results of indirect decompressions using intraoperative computed tomography after LLIF and analyzed the risk factors of insufficient indirect decompression resulting in unnecessary direct decompressions. In their study, two out of 28 patients required reoperation after lumbar decompression, but they did not include the revision surgery as a risk factor for insufficient indirect decompression [18]. Similar to our study, Hayama et al. [18] also found that the thecal sac was markedly enlarged after LLIF and that the rate of expansion was good enough to match those recorded in reports of primary surgeries. In addition, they found few perioperative complications similar to those observed in primary surgeries using LLIF, and they noted that the accompanying symptoms were only transient, similar to those observed in our study. They, as well as our group, found improvements in JOA scores after LLIF and after PLIF. Therefore, the clinical outcomes in revision cases may be considered good.

Regarding radiographical improvement on MRI, thecal sac area chronologically improved immediately after LLIF. Our previous study showed that the cross-sectional area of the thecal sac doubled 2 years after the primary surgery (excluding revision cases), and that the ligamentum flavum cross-sectional area and disk bulging both shrank significantly, although postoperative radiographical improvements do not appear to correlate with clinical symptoms [19]. Ohtori et al. [20] also reported a significant decrease in ligamentum flavum size 10 years after indirect decompression using anterior lumbar interbody fusion. Although there was operative scar tissue around the thecal sac in revision cases, the thecal sac continued to improve immediately after LLIF, just as in primary surgery cases. Scar tissue might also continue to decrease after surgery; however, further analysis based on larger-scale studies is necessary to confirm this.

There are several limitations in this study. First, it is possible that we may have underestimated the perioperative complication rates because of the retrospective nature of the study. Second, we conducted the study using a relatively small number of cases. In the future, large-scale prospective studies should be conducted to verify our findings. Third, the indications for surgery were different for PLIF and LLIF, and we excluded patients with severe preoperative neurological symptoms such as paralysis or severe leg pain at rest from the LLIF group because of the small number of such patients. Future studies will need to compare neurological improvement in cases of direct and indirect decompressions. Indirect decompression poses a risk of neurological deterioration after surgery in patients with severe neurological symptoms, including paralysis, and it should be noted that no surgical indications for 
such cases exist, even for primary operations. Thus, we believe that careful selection of patients for indirect decompression using LLIF is important.

\section{Conclusions}

In conclusion, indirect decompression using LLIF and PPS provides acceptable surgical outcomes and minimizes perioperative complications related to revision cases. Although revision procedures remain challenging for spine surgeons, indirect decompression could prove to be a relatively safe option allowing decompression regardless of the status of surgical scars.

\section{Conflict of Interest}

Tokumi Kanemura is a consultant for Medtronic and NuVasive. No potential conflict of interest relevant to this article was reported.

\section{Author Contributions}

Hiroaki Nakashima conducted analysis and interpretation of data. Tokumi Kanemura, Kotaro Satake, Hiroaki Nakashima, Jun Ouchida, Naoki Segi, and Hidetoshi Yamaguchi were surgeons in this study, and contributed in acquisition of data. Kenyu Ito supported the analysis and interpretation of data. Kenyu Ito and Shiro Imagama provided general support.

\section{References}

1. Rosenberg WS, Mummaneni PV. Transforaminal lumbar interbody fusion: technique, complications, and early results. Neurosurgery 2001;48:569-75.

2. Okuda S, Miyauchi A, Oda T, Haku T, Yamamoto T, Iwasaki M. Surgical complications of posterior lumbar interbody fusion with total facetectomy in 251 patients. J Neurosurg Spine 2006;4:304-9.

3. Tafazal SI, Sell PJ. Incidental durotomy in lumbar spine surgery: incidence and management. Eur Spine J 2005;14:287-90.

4. Cammisa FP Jr, Girardi FP, Sangani PK, Parvataneni HK, Cadag S, Sandhu HS. Incidental durotomy in spine surgery. Spine (Phila Pa 1976) 2000;25:2663-7.

5. Williams BJ, Sansur CA, Smith JS, et al. Incidence of unintended durotomy in spine surgery based on
108,478 cases. Neurosurgery 2011;68:117-24.

6. McMahon P, Dididze M, Levi AD. Incidental durotomy after spinal surgery: a prospective study in an academic institution. J Neurosurg Spine 2012;17:306.

7. Deyo RA, Martin BI, Kreuter W, Jarvik JG, Angier H, Mirza SK. Revision surgery following operations for lumbar stenosis. J Bone Joint Surg Am 2011;93:197986.

8. Ozgur BM, Aryan HE, Pimenta L, Taylor WR. Extreme lateral interbody fusion (XLIF): a novel surgical technique for anterior lumbar interbody fusion. Spine J 2006;6:435-43.

9. Oliveira L, Marchi L, Coutinho E, Pimenta L. A radiographic assessment of the ability of the extreme lateral interbody fusion procedure to indirectly decompress the neural elements. Spine (Phila Pa 1976) 2010;35(26 Suppl):S331-7.

10. Pawar AY, Hughes AP, Sama AA, Girardi FP, Lebl DR, Cammisa FP. A comparative study of lateral lumbar interbody fusion and posterior lumbar interbody fusion in degenerative lumbar spondylolisthesis. Asian Spine J 2015;9:668-74.

11. Fujibayashi S, Hynes RA, Otsuki B, Kimura H, Takemoto M, Matsuda S. Effect of indirect neural decompression through oblique lateral interbody fusion for degenerative lumbar disease. Spine (Phila Pa 1976) 2015;40:E175-82.

12. Elowitz EH, Yanni DS, Chwajol M, Starke RM, Perin NI. Evaluation of indirect decompression of the lumbar spinal canal following minimally invasive lateral transpsoas interbody fusion: radiographic and outcome analysis. Minim Invasive Neurosurg 2011;54:201-6.

13. Phillips FM, Isaacs RE, Rodgers WB, et al. Adult degenerative scoliosis treated with XLIF: clinical and radiographical results of a prospective multicenter study with 24-month follow-up. Spine (Phila Pa 1976) 2013;38:1853-61.

14. Isaacs RE, Hyde J, Goodrich JA, Rodgers WB, Phillips FM. A prospective, nonrandomized, multicenter evaluation of extreme lateral interbody fusion for the treatment of adult degenerative scoliosis: perioperative outcomes and complications. Spine (Phila Pa 1976) 2010;35(26 Suppl):S322-30.

15. Castellvi AE, Nienke TW, Marulanda GA, Murtagh RD, Santoni BG. Indirect decompression of lumbar 
stenosis with transpsoas interbody cages and percutaneous posterior instrumentation. Clin Orthop Relat Res 2014;472:1784-91.

16. Baghdadi YM, Larson AN, Dekutoski MB, et al. Sagittal balance and spinopelvic parameters after lateral lumbar interbody fusion for degenerative scoliosis: a case-control study. Spine (Phila Pa 1976) 2014;39:E166-73.

17. Pimenta L, Turner AW, Dooley ZA, Parikh RD, Peterson MD. Biomechanics of lateral interbody spacers: going wider for going stiffer. ScientificWorldJournal 2012;2012:381814.

18. Hayama S, Nakano A, Nakaya Y, et al. The evaluation of indirect neural decompression after lateral lumbar interbody fusion using intraoperative computed tomography myelogram. World Neurosurg 2018;120:e710-8.

19. Nakashima H, Kanemura T, Satake K, et al. Indirect decompression on MRI chronologically progresses after immediate post-lateral lumbar interbody fusion: the results from a minimum of 2 years follow-up. Spine (Phila Pa 1976) 2019 Jul 30 [Epub]. https://doi. org/10.1097/BRS.0000000000003180.

20. Ohtori S, Orita S, Yamauchi K, et al. Change of lumbar ligamentum flavum after indirect decompression using anterior lumbar interbody fusion. Asian Spine J 2017;11:105-12. 